\title{
Unfalsified Control Approach to Parameter Space Design of PID Controllers $^{\dagger}$
}

\author{
Masami SAEKI*
}

In this paper, unfalsified control concept is applied to the parameter space design problem of PID gains for mixed sensitivity control problem, and a new method of drawing unfalsified region on the parameter plane is proposed. This method does not require constructing a mathematical model for design, but draws the unfalsified region directly from the input-output responses of the plant. Therefore, it is expected that this method can be applied to complex nonlinear plants. By numerical experiments, it is found that sufficiently large region cannot be falsified by using a step response data, but can be falsified by using the responses of the plant excited by sinusoidal inputs for many frequencies. If the unfalsified region is empty, it can be concluded that no solution exist. Usefulness is demonstrated by a numerical example for a nonlinear plant with backlash.

Key Words: unfalsified control, PID control, parameter space design, mixed sensitivity problem, nonlinear plant

\section{Introduction}

When a plant contains nonlinear elements such as backlash, friction, and saturation, there is no design method that can be directly applied to such a complex plant ${ }^{12)}$. Therefore, unwillingly we approximate them by local linearization or describing function, or treat them as sector nonlinearities. Since this difficulty is fundamentally caused by our taking model-based design approach in control engineering, we can avoid it by developing a datadriven design method that does not require plant model. Unfalsified control concept proposed by Safonov ${ }^{1}$ ) is very interesting because it gives us such data-driven design methods. It has been applied to both adaptive con$\operatorname{trol}^{2), 3)}$, and off-line controller design ${ }^{4), 5 \text { ) }}$

On the other hand, the author has already proposed a parameter space design method of PID controllers for $H_{\infty}$ control problem and robust sensitivity control problem ${ }^{6) ~ 10)}$. These are convenient methods of drawing a necessary and sufficient solution set on the parameter plane. However, these methods use the frequency response of the plant, and therefore cannot be applied to nonlinear plants.

In the reference2), automatic tuning of PID controller is proposed for a mixed sensitivity performance index based on unfalsified concept, and it seems to be a promising

\footnotetext{
$\dagger$ Presented at SICE 3rd Annual Conference on Control Systems (2003.5)

* Faculty of Engineering, Hiroshima University, 1-4-1 Kagamiyama, Higashi-Hiroshima, Hiroshima

(Received April 17, 2003)

(Revised December 8, 2003)
}

approach for parameter space design if the following difficulties are got rid of.

a) In the unfalsified approach, a finite number of candidate controllers are prepared and some of them will be falsified adaptively by the input-output data. However, we want to treat infinite number of PID gains on the parameter plane.

b) Unfalsified adaptive controller makes use of the inverse of the controller. But we cannot obtain a proper algorithm for drawing the solution set because of the inversion, and it is necessary to avoid it.

c) Relation between the largeness of the falsified region and the class of input-output data has not been examined in the previous studies, and it is important to find a class of input-output data that make the falsified region as large as possible.

In the reference 5), the first two problems are cleared for an $l_{\infty}$ norm performance index and a class of linear timeinvariant controllers, and the design problem is reduced to a linear programming problem.

In this paper, we will propose a parameter space design method of PID controllers for a mixed sensitivity performance index via unfalsified control approach.

\section{Problem formulation}

Let us consider the feedback system described by

$$
\begin{aligned}
& y=P e \\
& u=K y \\
& e=w-u
\end{aligned}
$$

where $P$ is a single-input single-output plant whose property is defined by a set of input output data, and $K$ is 


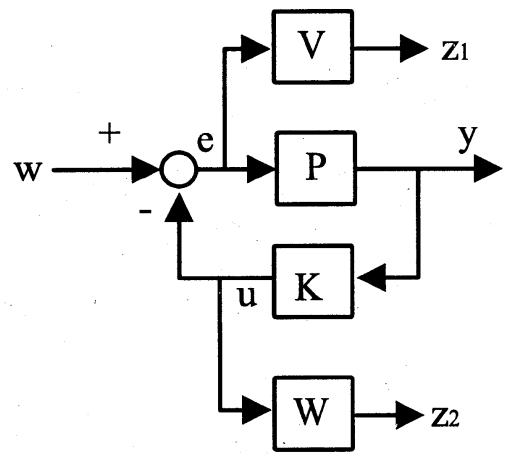

Fig. 1 Mixed sensitivity problem

a controller. $y, u, e, w$ are discrete time signals with sampling time $\Delta T$ where $u=\{u(1), u(2), \ldots\}$ and other signals are defined in the same way. We assume that the input output data $e(i), y(i)$ of the plant are given where the initial condition of the plant is zero. The controller $K$ is a discrete time PID controller of the form

$$
K\left(z^{-1}\right)=p_{1}+p_{2} \frac{z^{-1}}{1-z^{-1}}+p_{3}\left(1-z^{-1}\right)
$$

Introduce weighting functions $V\left(z^{-1}\right), W\left(z^{-1}\right)$ and new variables

$$
z_{1}=V e, z_{2}=W u
$$

as shown in Fig. 1, and our design specification is given by the $l_{2}$-gain condition: for all $w \in l_{2}$

$$
\|z\|_{2}<\gamma\|w\|_{2}
$$

where $z=\left(z_{1}, z_{2}\right)^{T}$ and

$$
\begin{aligned}
& \|z\|_{2}=\sqrt{\sum_{i=1}^{\infty}\left\{z_{1}^{2}(i)+z_{2}^{2}(i)\right\}} \\
& \|w\|_{2}=\sqrt{\sum_{i=1}^{\infty} w^{2}(i)}
\end{aligned}
$$

Our problem is to obtain such PID gains $p_{1}, p_{2}, p_{3}$ that do not satisfy $(6)$.

Remark 1 If the plant were linear time-invariant with a transfer function $P\left(z^{-1}\right)$, the $l_{2}$-gain condition $(6)$ is equivalent to a mixed sensitivity control condition described by the $H_{\infty}$ norm condition.

$$
\left\|\left(\begin{array}{c}
V S \\
W T
\end{array}\right)\right\|_{\infty}<\gamma
$$

where the sensitivity function $S\left(z^{-1}\right)$ and the complementary sensitivity function $T\left(z^{-1}\right)$ are given by

$$
S=\frac{1}{1+P K}, \quad T=\frac{P K}{1+P K}
$$

In this case, namely when the plant is linear timeinvariant and the frequency response is available, a necessary and sufficient region of PID gains that satisfy (9) can be drawn on the parameter plane by the frequency domain method of the references 8) and 9).

\section{Falsification condition}

Lemma 1 (Theorem 2.1 of the reference 11)) Let $F$ be a causal operator from the extended space $W_{e}$ into itself. If $F$ is bounded on $W_{e}$, then $F$ maps $W$ into itself and the bounds of $F$ on $W_{e}$ and $W$ are equal. Conversely, if $F$ maps $W$ into itself and is bounded on $W$, then $F$ is bounded on $W_{e}$ and the bounds of $F$ on $W$ and $W_{e}$ are equal.

This property has already been used to derive a falsification condition in the reference 1). By using this property, we will give a new unfalsification condition suitable for parameter space design of PID gain.

Represent the integral and derivative values of $y$ as

$$
y_{I}=\frac{z^{-1}}{1-z^{-1}} y, \quad y_{D}=\left(1-z^{-1}\right) y
$$

and the responses of $W$ to $y, y_{I}, y_{D}$ as

$$
y_{W}=W y, \quad y_{W I}=W y_{I}, \quad y_{W D}=W y_{D}
$$

Then, put

$$
\begin{aligned}
& v_{1}(i)=\left[y_{W}(i), y_{W I}(i), y_{W D}(i)\right]^{\mathrm{T}} \\
& v_{2}(i)=\left[y(i), y_{I}(i), y_{D}(i), e(i)\right]^{\mathrm{T}}
\end{aligned}
$$

and define the following matrices where $c_{1} \in R, R \in$ $R^{3 \times 3}, Q \in R^{3 \times 3}, q \in R^{3 \times 1}, c_{2} \in R$.

$$
\begin{aligned}
c_{1}(m) & :=\sum_{i=1}^{m} z_{1}^{2}(i) \\
R(m) & :=\sum_{i=1}^{m} v_{1}(i) v_{1}(i)^{\mathrm{T}} \\
\left(\begin{array}{cc}
Q(m) & q(m) \\
q^{\mathrm{T}}(m) & c_{2}(m)
\end{array}\right): & =\sum_{i=1}^{m} v_{2}(i) v_{2}(i)^{\mathrm{T}}
\end{aligned}
$$

Theorem 1 The $l_{2}$ gain condition (6) is satisfied for all $w \in l_{2}$, if and only if

$$
\begin{array}{r}
p^{\mathrm{T}}\left[Q(m)-\frac{1}{\gamma^{2}} R(m)\right] p+2 p^{\mathrm{T}} q(m)+c_{2}(m) \\
-\frac{1}{\gamma^{2}} c_{1}(m)>0, m=1,2, \ldots
\end{array}
$$

is satisfied for all $w \in l_{2 e}$ where $p$ is a parameter vector given by

$$
p=\left(p_{1}, p_{2}, p_{3}\right)^{\mathrm{T}}
$$

proof) From Lemma 1 , the $l_{2}$ gain condition (6) is satisfied, if and only if

$$
\|z\|_{2[1, m]}<\gamma\|w\|_{2[1, m]}, m=1,2, \ldots
$$


is satisfied for all $w \in l_{2 e}$ where $\|w\|_{2[1, m]}$ denotes the $l_{2}$ norm of the truncated signal;

$$
\|w\|_{2[1, m]}=\sqrt{\sum_{i=1}^{m} w^{2}(i)}
$$

Next, let us represent (18) by using the parameter vector $p$. Substitution of $z_{2}=W K y$ and $w=K y+e$ into (18) gives

$$
\begin{array}{r}
\left\|z_{1}\right\|_{2[1, m]}^{2}+\|W K y\|_{2[1, m]}^{2} \\
<\gamma^{2}\|K y+e\|_{2[1, m]}^{2}
\end{array}
$$

Since

$$
\begin{aligned}
K y & =p_{1} y+p_{2} y_{I}+p_{3} y_{D} \\
W K y & =p_{1} y_{W}+p_{2} y_{W I}+p_{3} y_{W D}
\end{aligned}
$$

, each term is represented as

$$
\begin{aligned}
& \left\|z_{1}\right\|_{2[1, m]}^{2}=c_{1}(m) \\
& \|W K y\|_{2[1, m]}^{2}=p^{\mathrm{T}} R(m) p \\
& \|K y+e\|_{2[1, m]}^{2} \\
& =\left(\begin{array}{cc}
p^{\mathrm{T}} & 1
\end{array}\right)\left(\begin{array}{cc}
Q(m) & q(m) \\
q^{\mathrm{T}}(m) & c_{2}(m)
\end{array}\right)\left(\begin{array}{l}
p \\
1
\end{array}\right)
\end{aligned}
$$

Substituting these terms into (20) gives (16) immediately. This completes the proof.

Remark 2 Note that controller inversion does not appear in the above derivation. If we reverse the order of the plant and the controller of the feedback system in Fig. 1, which is used in the reference 1 ), a fictitious reference input $w$ is given by $w=y+K^{-1} u$. In this case, $K$ must be causally left invertible and a falsification condition suitable for drawing the solution set cannot be derived.

Remark 3 The region that satisfies (16) can be drawn on the parameter plane in the following way. Suppose that the plane is given by $p_{3}=a$. First, substitute $p=\left(p_{1}, p_{2}, a\right)^{T}=\left(\tilde{p}^{T}, a\right)^{T}$ into (16) and represent it in the next form

$$
\tilde{p}^{T} \tilde{Q} \tilde{p}+2 \tilde{p}^{T} \tilde{q}(m)+\tilde{c}(m)>0
$$

where $\tilde{p}=\left(p_{1}, p_{2}\right)^{T}$. Then draw the falsified region by the algorithm given in Appendix. The shape of the region will be ellipse or hyperbola depending on the sign of the eigenvalues $\lambda_{1}, \lambda_{2}$ of the matrix $Q$, if $\lambda_{1}, \lambda_{2}$ are nonzero. If $\lambda_{1}=0$ and $\lambda_{2}=0$, the region is a half plane. If one of $\lambda_{i}$ is zero, the boundary of the region is a parabola.

\section{Input-output data selection}

In this section, we will examine a proper class of inputoutput data that makes the falsified region larger by a numerical example. Let us consider the system shown in

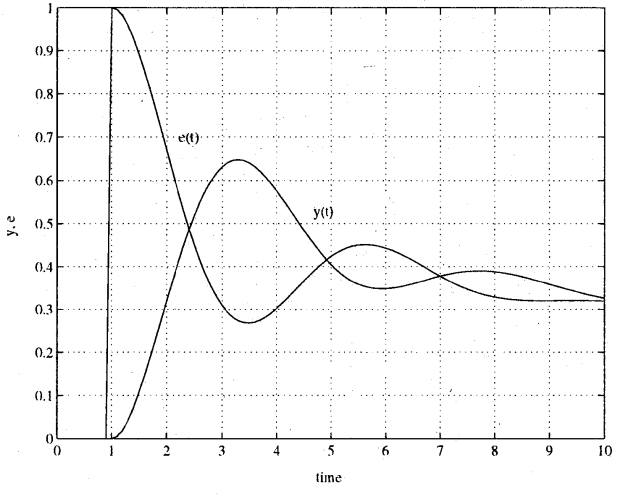

Fig. 2 Step response $y(t)$ and $e(t)$

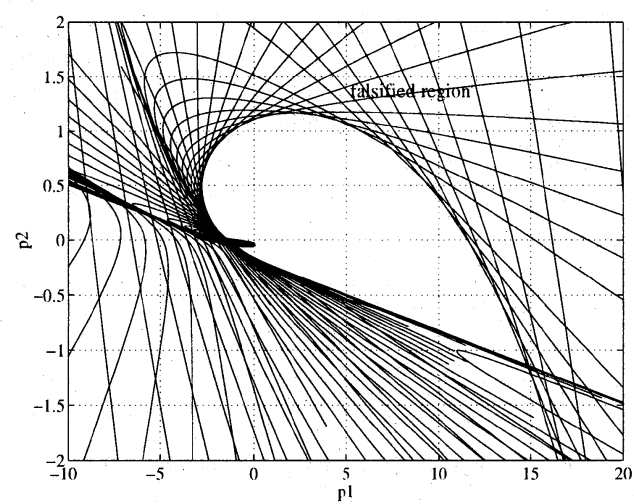

Fig. 3 Falsified region by data-driven method (a step response data)

Fig. 1 where

$$
\begin{array}{r}
P(s)=\frac{1}{s^{2}+s+1}, K(s)=1+\frac{0.01}{s} \\
V(s)=\frac{100 s+1}{255 s}, W(s)=\frac{0.5 s+1}{1.5}
\end{array}
$$

The unit step response $e(t), y(t)$ is obtained by Simulink in the interval $[0,20][\mathrm{sec}]$ with the sampling time $\Delta T=$ 0.05[sec] as shown in Fig. 2. Then, by using the data, the falsified regions determined by (16) with $\gamma=1$ are drawn on the parameter plane in Fig. 3 for $m=1,2, \ldots, 400$.

On the other hand, since the plant is linear timeinvariant, the exact region can be obtained by the frequency domain method ${ }^{8)}$ if the plant transfer function is supposed to be known. Discretization of $P(s), V(s), W(s)$ with the sampling time $\Delta T=0.05$ gives

$$
\begin{aligned}
P_{z}\left(z^{-1}\right) & =\frac{0.001229 z+0.001209}{z^{2}-1.949 z+0.9512} \\
V_{z}\left(z^{-1}\right) & =\frac{0.3923 z-0.3921}{z-1} \\
W_{z}\left(z^{-1}\right) & =\frac{14 z-12.67}{z+1}
\end{aligned}
$$

and the exact region is shown in Fig. 4.

The unfalisified region in Fig. 3 is about ten times larger than the exact region in Fig. 4. We have also tested for sawtooth and random reference inputs, but the unfalsified 


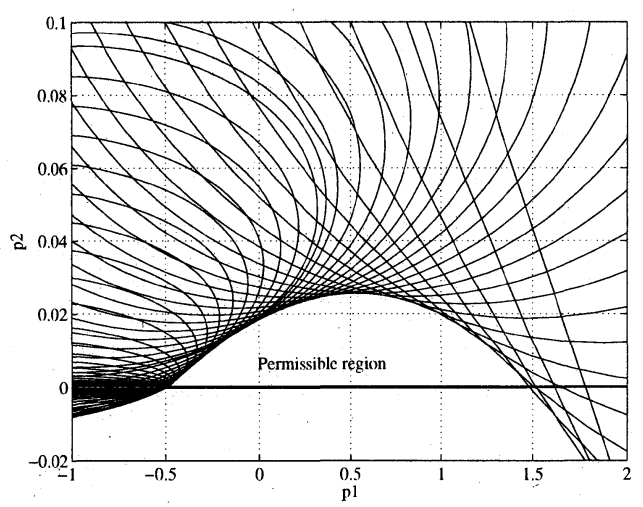

Fig. 4 Exact region by frequency domain method

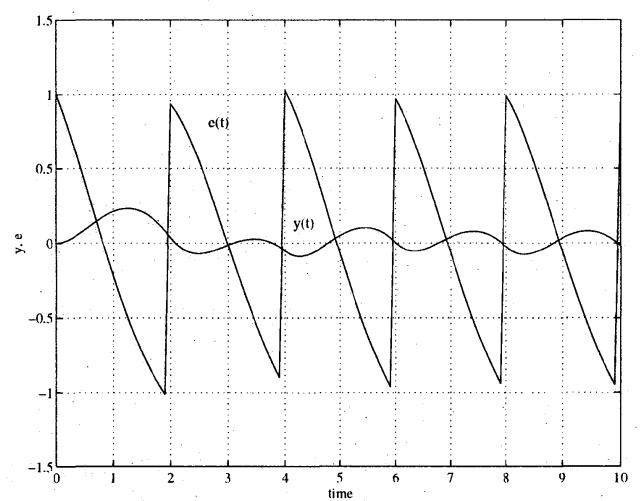

Fig. 5 Response $y(t)$ and $e(t)$ for saw reference input

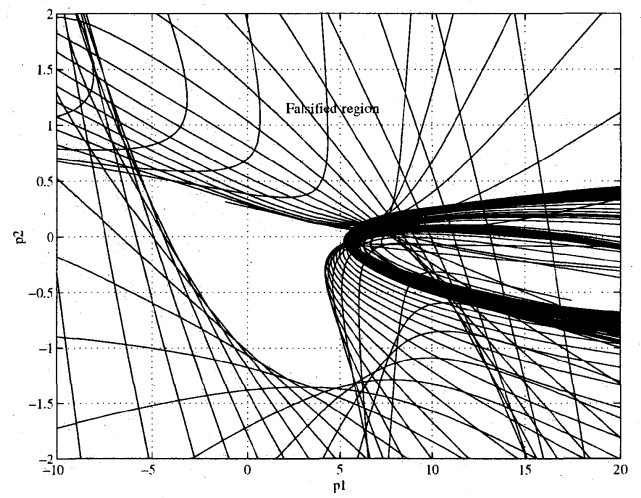

Fig. 6 Falsified region by data-driven method (a saw response data)

regions are much larger than the exact region similarly. The sawtooth case is shown in Fig. 5 and Fig. 6. This numerical experiment suggests that the region which can be falsified by truncating a single input-output data may be not large enough. Note that this does not deny the adaptive method where a single data is also used in real time, because the PID gain is fixed in our method but changed adaptively in the tuning method.

In order to overcome this difficulty, we will use sinusoidal inputs with many frequencies $w(t)=\sin \omega t$. We cannot prove this data selection works well for nonlinear systems generally, but at least we may justify the selection for linear time-invariant stable systems as follows.

Let us consider a system $G(s) \in R H_{\infty}$. Then, the response $z(t) \in R^{q}$ of the system for the input $w(t)=$ $\sin \omega t, t \geq 0$ satisfies

$$
z(t) \rightarrow z_{\infty}(t)=A \sin (\omega t+\phi) \quad(t \rightarrow \infty)
$$

where $A=|G(j \omega)|$ and $\phi=\angle G(j \omega)$, and the error $e(t)=z(t)-z_{\infty}(t)$ is bounded by

$$
|e(t)|<M e^{-\alpha t}
$$

for certain positive constants $M$ and $\alpha$. On this condition, the next theorem is satisfied.

Theorem 2 For a giveh $\delta>0$,

$$
\frac{\sqrt{\int_{0}^{T} z^{2}(t) d t}}{\sqrt{\int_{0}^{T} w^{2}(t) d t}} \leq|G(j \omega)|[1+\delta]
$$

is satisfied for a sufficiently large $T$ that satisfies

$$
T>\frac{1}{2 \omega}+\frac{2}{2 \delta+\delta^{2}}\left(\frac{1}{\omega}+\frac{1}{\alpha A^{2}}\left[2 M A+\frac{M^{2}}{2}\right]\right)
$$

Proof)

$$
\begin{aligned}
& \int_{0}^{T} z^{2}(t) d t= \\
& \int_{0}^{T}\left\{z_{\infty}^{2}(t)+2 e(t) z_{\infty}(t)+e^{2}(t)\right\} d t \\
& \leq A^{2} \int_{\phi / \omega}^{T+\phi / \omega} \sin ^{2} \omega \hat{t} d \hat{t}+2 M A\left(\frac{1}{\alpha}-\frac{1}{\alpha} e^{-\alpha T}\right) \\
& +M^{2}\left(\frac{1}{2 \alpha}-\frac{1}{2 \alpha} e^{-2 \alpha T}\right) \\
& \leq A^{2} \int_{0}^{T} w^{2}(t) d t+\frac{A^{2}}{\omega}+\frac{1}{\alpha}\left(2 M A+\frac{M^{2}}{2}\right)
\end{aligned}
$$

Further,

$$
\int_{0}^{T} w^{2}(t) d t=\frac{T}{2}-\frac{1}{4 \omega} \sin 2 \omega T
$$

The left-hand side of (29) is bounded as

$$
\frac{\sqrt{\int_{0}^{T} z^{2}(t) d t}}{\sqrt{\int_{0}^{T} w^{2}(t) d t}} \leq A \sqrt{1+\frac{\frac{1}{\omega}+\frac{1}{\alpha A^{2}}\left(2 M A+\frac{M^{2}}{2}\right)}{\frac{T}{2}-\frac{1}{4 \omega} \sin 2 \omega T}}(32)
$$

The condition that the right-hand side of (32) is smaller than $A(1+\delta)$ gives (30). This completes the proof.

This theorem implies that the time domain condition $\int_{0}^{T} z^{2}(t) d t<\gamma^{2} \int_{0}^{T} w^{2}(t) d t$ comes closer to the frequency domain condition $|G(j \omega)|<\gamma, \omega \in R$ for a sufficiently large $T$. Therefore, the condition (16) is almost equal to the frequency domain condition for a sufficiently large $m$, namely $m=n$. Therefore we may only draw the region of (16) for $m=n$ instead of $m=1, \ldots, n$ for each frequency.

By applying this method to the above numerical example for 50 frequencies[rad/sec] logarithmically equally 


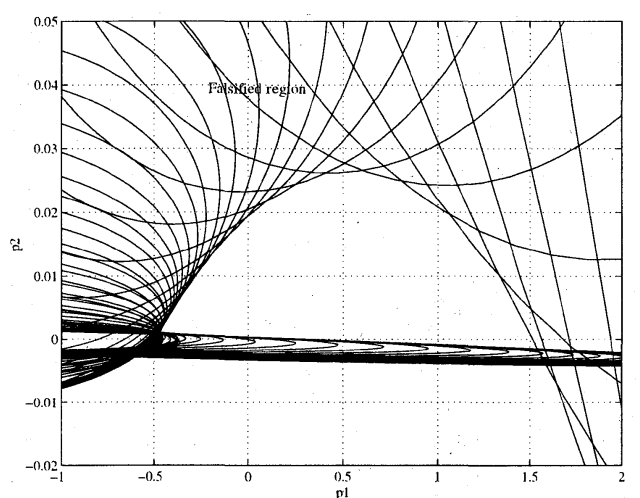

Fig. 7 Falsified region by data-driven method (many sinusoidal reference inputs)

spaced between 0.0001 and 10 , we obtain the falsified region as shown in Fig. 7. Each curve corresponds to each frequency and the falsified region is very close to the exact region.

Let us explain a procedure of finding a candidate PID gain. As an example, we try to make $\gamma$ of (6) smaller.

\section{Design procedure}

(1) Obtain $e(k \Delta T)$ and $y(k \Delta T), k=1, \ldots, n$ for the sinusoidal inputs $w(t)=A \sin \left(\omega_{i} t\right), i=1, \ldots, N$ with the initial condition of the plant zero for a feedback system in Fig. 1. $\omega_{i}$ 's are $N$ frequencies logarithmically equally spaced between $10^{d_{1}}$ and $10^{d_{2}}$. The amplitude $A$ may be determined by the range of the responses.

(2) Choose $p_{3}=a$ (at first, usually $a=0$ ) and draw the falsified region that does not satisfy (16) for $m=n$ on $\left(p_{1}, p_{2}, a\right)$ plane for each frequency following Remark 3. The unfalsified region will shrink by setting $\gamma$ smaller.

(3) Choose the value of $p_{2}=b$ from the unfalsified region, and draw the falsified region on $\left(p_{1}, b, p_{3}\right)$ plane for each frequency. The unfalsified region will shrink by setting $\gamma$ smaller.

(4) After having iterated the above steps (2) and (3), we choose a candidate controller.

Remark 4 It is easier to get the data experimentally for mechanical systems than chemical processes. Even for mechanical systems, it is desirable to decrease the amount of data. Since the curves that form the boundary of unfalsified region is not so many as shown in Fig. 7 , we can expect that it will be decreased. On the other hand, if we have a detailed model for simulation, our method can be applied to it without constructing a simple model for controller design.

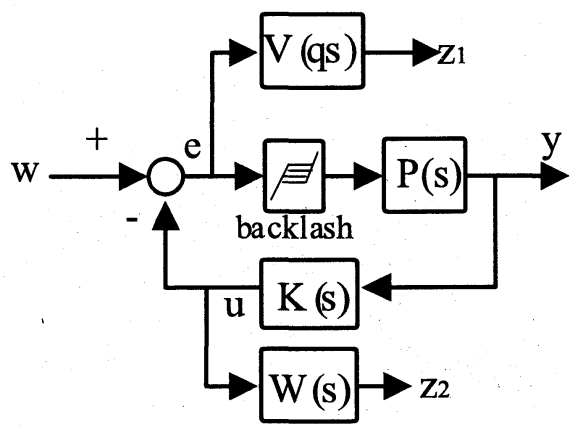

Fig. 8 Mixedsensitivity problem for a nonlinear plant

\section{Numerical example : nonlinear plant case}

Let us consider the nonlinear system with the performance weights as shown in Fig. 8 where a backlash nonlinearity with the width one is inserted at the plant input and $V(q s)=\frac{100 q s+1}{255 q s}$ is used instead of $V(s)$ for the tuning of the sensitivity weight. We examine the minimization of $q$ with $\gamma=1$.

Unfalsified region shrinks as $q$ decreases from 1 as shown in Fig. 9, 10, 11, and finally it disappears at $q=0.234$ for $p_{1}=0.38, p_{2}=0.028$. This means that no PI gain make $q$ smaller than $q=0.234$. If the backlash is removed, the unfalsified region disappears at $q=0.0119$ for $p_{1}=0.55, p_{2}=0.0225$. Increase of $q$ from 0.0119 to 0.234 suggests that the backlash deteriorates the performance. The step responses are shown in Fig. 12 where

a) Plant is linear and the controller is

$$
K_{1}\left(z^{-1}\right)=0.55+\frac{0.0225}{1-z^{-1}}
$$

b) Plant contains backlash and the controller is $K_{1}\left(z^{-1}\right)$,

c) Plant contains backlash and the controller is

$$
K_{2}\left(z^{-1}\right)=0.38+\frac{0.028}{1-z^{-1}}
$$

\section{Conclusion}

In this paper, a design method of PID controllers on the parameter plane is proposed for a mixed sensitivity problem. Since the cost function can be expressed in terms of quadratic inequalities (ellipses and hyperbolas) in the PID parameter vector, the falsified region can be drawn easily on the parameter plane. An appropriate input-output data set that gives a larger estimate of the falsified region is examined by simulation, and it is found that a single step response data does not give a sufficiently large estimate, but a set of data given by sinusoidal reference inputs does. This method may be suitable for those mechanical systems to which a vibration test can be applied. 


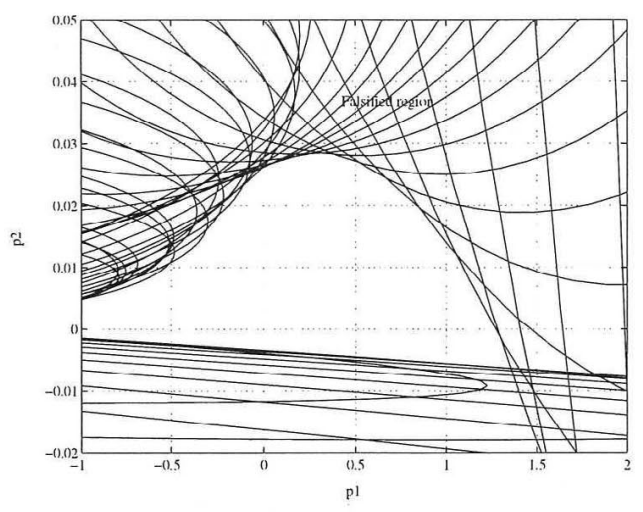

Fig. 9 Parameter plane for $q=0.5$

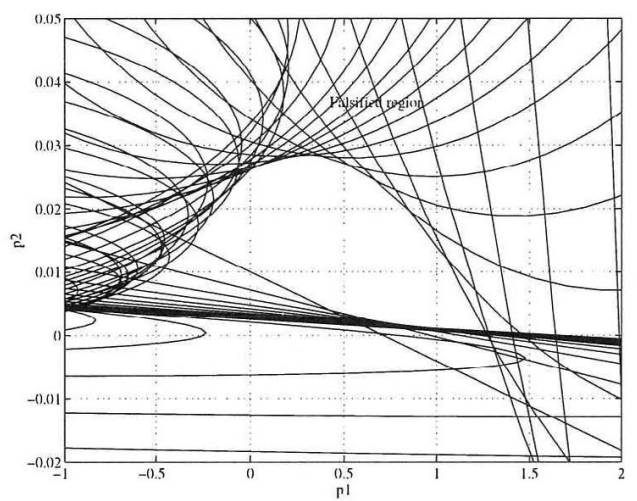

Fig. 10 Parameter plane for $q=0.25$

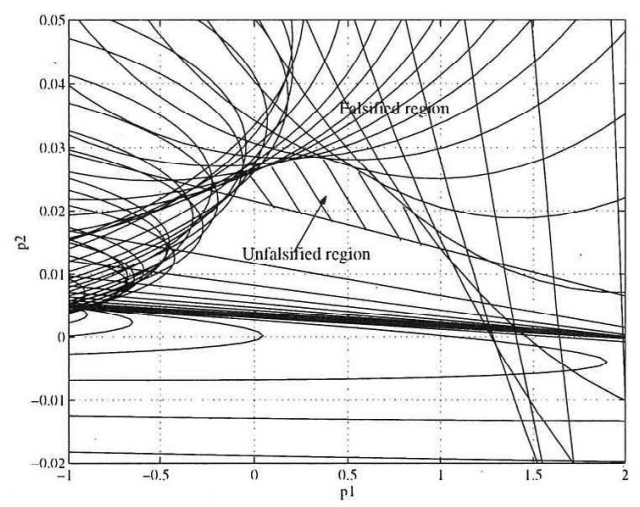

Fig. 11 Parameter plane for $q=0.24$

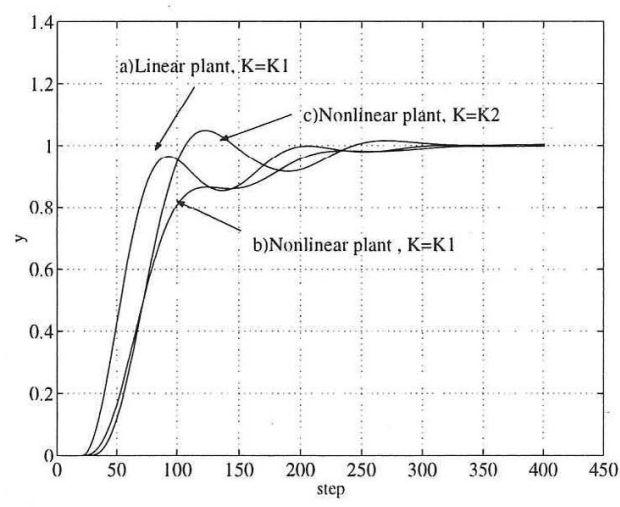

Fig. 12 Step responses
Moreover, if we already have a detailed model for simulation, we can apply our method directly.

Acknowledgement: The author would like to thank Professor Safonov for his useful comments on Lemma 1.

\section{References}

1) M.G. Safonov and T.C. Tsao: The unfalsified control concept and learning. IEEE Trans. Automat. Contr., AC-42$6,843 / 847$ (1997)

2) M. Jun and M.G. Safonov: Automatic PID tuning: An application of unfalcificd control. Proc. of the 1999 IEEE International Symposium on Computer Aided Control System Design, 328/333 (1999)

3) T.C. Tsao and M.G. Safonov: Unfalsified direct adaptive control of a two-link robot arm. International Journal of Adaptive Control and Signal Processing, 15, 319/334 (2001)

4) E.G. Collins and C. Fan: Automated PI tuning for a weight belt feeder via unfalsified control. Proc. of the 38th Conference on Decision \& Control, 785/790 (1999)

5) B.R. Woodley, J.P. How and R.L. Kosut: Direct unfalsified controller design - Solution via convex optimization. Proc. of the American Control Conference, 3302/3306 (1999)

6) M. Saeki: A design method of the optimal PID controller for a two disc type mixed sensitivity problem. Trans. of the Inst. of Sys. Contr. Info. Eng., 7-12, 520/527 (1994)

7) M. Saeki and D. Hirayama: Parameter space design method of PID controller for robust sensitivity minimization problem. Trans. of the Society of Instrument and Control Engineers, 32-12, 1612/1619 (1996)

8) M. Saeki and J. Kimura: Design method of robust PID controller and CAD system. 11th IFAC Symposium on System Identification, 3, 1587/1593 (1997)

9) M. Saeki, J. Kimura, Y. Kurata and S. Taiji: Parameter space design method of PID controller for $H_{\infty}$ control problem. Trans. of the Inst. of Sys. Contr. Info. Eng., 11$1,35 / 40(1998)$

10) M. Saeki and K. Aimoto: PID controller optimization for $H_{\infty}$ control by linear programming. International Journal of Robust Nonlinear Control, 10, 83/99 (2000)

11) J.C. Willems: The Analysis of Feedback Systems, THE M.I.T. PRESS, p.15 (1971)

12) M. Nordin and P.O. Gutman: Controlling mechanical systems with backlash-a survey. Automatica, 38, 1633/1649 (2002)

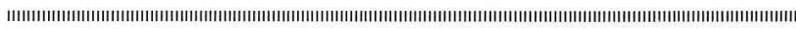

\section{Masami Saeki (Member)}

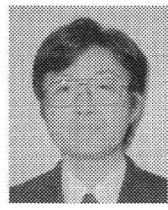

He received the B.E., M.E. and Ph.D degrees from Kyoto University in 1976, 1978 and 1982, respectively. Since 1992, he has been a Professor at the Faculty of Engineering, Hiroshima University. His research interests include robust control design and its application. He is a member of ISCIE, JSME and IEEE. 


\section{Appendix}

We will summarize how to draw the region $\Phi$ defined by

$$
\Phi=\left\{p \in R^{2} \mid p^{T} Q p+2 p^{T} q+r>0\right\}
$$

Decompose the symmetric matrix $Q$ as

$$
Q=U^{T} \Lambda U, \Lambda=\operatorname{diag}\left(\lambda_{1}, \lambda_{2}\right)
$$

and represent (33) as

$$
x^{T} \Lambda x+2 x^{T} \hat{q}+r>0
$$

where $x=U^{T} p$ and $\hat{q}=U^{T} q$.

In the following, put $c_{0}=\hat{q}^{T} \Lambda^{-1} \hat{q}-r$.

(1) $\lambda_{1}>0, \lambda_{2}>0$

(a) $c_{0}<0$, then all $p$

(b) $c_{0}>0$, then outside the ellipse given by

$$
p=U \sqrt{c_{0}} \Lambda^{-\frac{1}{2}} y-U \Lambda^{-1} \hat{q}
$$

where $y=(\sin \theta, \cos \theta)^{T}$ for $0 \leq \theta \leq 2 \pi$

(2) $\lambda_{1}<0, \lambda_{2}<0$

(a) $c_{0}>0$, then empty set.

(b) $c_{0}<0$, then inside the ellipse given by

$$
p=U \sqrt{-c_{0}}(-\Lambda)^{-\frac{1}{2}} y-U \Lambda^{-1} \hat{q}
$$

where $y=(\sin \theta, \cos \theta)^{T}$ for $0 \leq \theta \leq 2 \pi$

(3) $\lambda_{1}>0, \lambda_{2}<0$

(a) $c_{0}>0$, outer region of the hyperbolic defined by

$$
\begin{array}{r}
p=U \sqrt{c_{0}} \operatorname{diag}\left(\lambda_{1}^{-\frac{1}{2}},\left(-\lambda_{2}\right)^{-\frac{1}{2}}\right) y \\
-U \Lambda^{-1} \hat{q}
\end{array}
$$

where $y=\frac{ \pm 1}{\sqrt{\cos ^{2} \theta-\sin ^{2} \theta}}(\cos \theta, \sin \theta)^{T}$ for $-\pi / 4<\theta<$ $\pi / 4$.

(b) $c_{0}<0$, inner region of the hyperbolic defined by

$$
\begin{array}{r}
p=U \sqrt{-c_{0}} \operatorname{diag}\left(\lambda_{1}^{-\frac{1}{2}},\left(-\lambda_{2}\right)^{-\frac{1}{2}}\right) y \\
-U \Lambda^{-1} \hat{q}
\end{array}
$$

where $y=\frac{ \pm 1}{\sqrt{\sin ^{2} \theta-\cos ^{2} \theta}}(\cos \theta, \sin \theta)^{T}$ for $\frac{1}{4} \pi<\theta<$ $\frac{3}{4} \pi$.

(4) $\lambda_{1}<0, \lambda_{2}>0$

(a) $c_{0}>0$, outer region of the hyperbolic defined by

$$
\begin{array}{r}
p=U \sqrt{c_{0}} \operatorname{diag}\left(\left(-\lambda_{1}\right)^{-\frac{1}{2}},\left(\lambda_{2}\right)^{-\frac{1}{2}}\right) y \\
-U \Lambda^{-1} \hat{q}
\end{array}
$$

where $y=\frac{ \pm 1}{\sqrt{\cos ^{2} \theta-\sin ^{2} \theta}}(\cos \theta, \sin \theta)^{T}$ for $\frac{1}{4} \pi<\theta<$ $\frac{3}{4} \pi$.

(b) $c_{0}<0$, inner region of the hyperbolic defined by

$$
\begin{array}{r}
p=U \sqrt{-c_{0}} \operatorname{diag}\left(\left(-\lambda_{1}\right)^{-\frac{1}{2}},\left(\lambda_{2}\right)^{-\frac{1}{2}}\right) y \\
-U \Lambda^{-1} \hat{q}
\end{array}
$$

where $y=\frac{ \pm 1}{\sqrt{\sin ^{2} \theta-\cos ^{2} \theta}}(\cos \theta, \sin \theta)^{T}$ for $-\frac{1}{4} \pi<\theta<$ $\frac{1}{4} \pi$.

The case of $\lambda_{1} \lambda_{2}=0$ is given in the following, though it does not occur in generic.

(1) $\lambda_{1}=0, \lambda_{2}=0$

The unfalsified region is a half-plane given by

$$
2 p^{T} \hat{q}+r>0
$$

(2) $\lambda_{2}=0$

(a) $\hat{q}_{2} \neq 0$

The boundary of the region, a parabola, is given by $p=U\left(x_{1}, x_{2}\right)^{T}$ and

$$
\begin{aligned}
& x_{1}=X-\frac{\hat{q}_{1}}{\lambda_{1}} \\
& x_{2}=-\frac{\lambda_{1}}{2 \hat{q}_{2}} Y-\left(r-\frac{\hat{q}_{1}^{2}}{\lambda_{1}}\right) \frac{1}{2 \hat{q}_{2}}
\end{aligned}
$$

where $X$ and $Y$ satisfy $Y=X^{2}$. If $\lambda_{1}>0$, the unfalsified region is outside the parabola. If $\lambda_{1}<0$, the unfalsified region is inside the parabola.

(b) $\hat{q}_{2}=0$

If $\lambda_{1}>0$ and $D_{1}=\hat{q}_{1}^{2}-\lambda_{1} r<0$, all points are unfalsified. If $\lambda_{1}>0$ and $D_{1} \geq 0, x_{1} \leq \underline{x}_{1}$ or $x_{1} \geq \bar{x}_{1}$ where

$$
\underline{x}_{1}=\frac{-\hat{q}_{1}-\sqrt{D_{1}}}{\lambda_{1}}, \bar{x}_{1}=\frac{-\hat{q}_{1}+\sqrt{D_{1}}}{\lambda_{1}}
$$

If $\lambda_{1}<0$ and $D_{1}<0$, all points are falsified. If $\lambda_{1}<0$ and $D_{1} \geq 0, \underline{x}_{1} \leq x_{1} \leq \bar{x}_{1}$.

(3) $\lambda_{1}=0$

(a) $\hat{q}_{1} \neq 0$

The boundary of the region, a parabola, is given by $p=U\left(x_{1}, x_{2}\right)^{T}$ and

$$
\begin{aligned}
& x_{1}=-\frac{\lambda_{2}}{2 \hat{q}_{1}} Y-\left(r-\frac{\hat{q}_{2}^{2}}{\lambda_{2}}\right) \frac{1}{2 \hat{q}_{1}} \\
& x_{2}=X-\frac{\hat{q}_{2}}{\lambda_{2}}
\end{aligned}
$$

where $X$ and $Y$ satisfy $Y=X^{2}$. If $\lambda_{2}>0$, the unfalsified region is outside the parabola. If $\lambda_{2}<0$, the unfalsified region is inside the parabola.

(b) $\hat{q}_{1}=0$

If $\lambda_{2}>0$ and $D_{2}=\hat{q}_{2}^{2}-\lambda_{2} r<0$, all points are unfalsified. If $\lambda_{2}>0$ and $D_{2} \geq 0, x_{2} \leq \underline{x}_{2}$ or $x_{2} \geq \bar{x}_{2}$ where

$$
\underline{x}_{2}=\frac{-\hat{q}_{2}-\sqrt{D_{2}}}{\lambda_{2}}, \bar{x}_{2}=\frac{-\hat{q}_{2}+\sqrt{D_{2}}}{\lambda_{2}}
$$

If $\lambda_{2}<0$ and $D_{2}<0$, all points are falsified: If $\lambda_{2}<0$ and $D_{2} \geq 0, \underline{x}_{2} \leq x_{2} \leq \bar{x}_{2}$. 